\title{
Philosophiques
}

\section{Sur les justifications de la philosophie institutionnalisée}

\section{Jean-Paul Brodeur}

Volume 3, numéro 1, avril 1976

URI : https://id.erudit.org/iderudit/203048ar

DOI : https://doi.org/10.7202/203048ar

Aller au sommaire du numéro

Éditeur(s)

Société de philosophie du Québec

ISSN

0316-2923 (imprimé)

1492-1391 (numérique)

Découvrir la revue

Citer ce document

Brodeur, J.-P. (1976). III. Sur les justifications de la philosophie

institutionnalisée. Philosophiques, 3(1), 109-122.

https://doi.org/10.7202/203048ar d'utilisation que vous pouvez consulter en ligne.

https://apropos.erudit.org/fr/usagers/politique-dutilisation/ 


\title{
III. SUR LES JUSTIFICATIONS DE LA PHILOSOPHIE INSTITUTIONNALISÉE
}

\author{
par Jean-Paul Brodeur
}

Préambule. Le but de ma communication au colloque sur l'institutionnalisation de la philosophie était de fournir une brève analyse des diverses raisons que l'on a présentées pour justifier la persistance au sein des curricula officiels de périodes dévolues à l'enseignement de la philosophie. J'aimerais, avant de présenter mon analyse, faire deux remarques sur le discours de la justification.

Le discours de justification, surtout lorsqu'il a pour objet de montrer l'opportunité de continuer d'institutionnaliser la pratique d'une discipline à l'intérieur de l'école, appartient au registre pratique du discours. Le discours pratique se manifeste à nous avec les traits suivants: (i) il est d'abord profondément circonstancié, étant par nature attentif aux particularités d'une conjoncture; (ii) il est ensuite enclin à utiliser les ressources de la rhéthorique, ayant pour fonction première de convaincre. A cause de la présence de ces deux traits, tenir un discours de justification est une activité relativement ingrate qu'il est par trop facile de dévaluer en la mesurant à l'aune de critères 
qui valent avant tout du discours théorique, à savoir l'universalité des propositions et l'absence d'emphase. Il me semble que l'on devrait se garder de céder à la tentation des réfutations aisées.

Cette mise en garde demeure souvent lettre morte, les congrès sur l'institutionnalisation de la philosophie se scindant la plupart du temps en deux groupes, dont l'un, se percevant comme celui des chercheurs ou des théoriciens, s'occupe à faire des remontrances à l'autre. Peut-on, en s'autorisant de notre première remarque, rendre compte de ces affrontements récurrents par un défaut d'empathie des chercheurs (ou de ceux qui se perçoivent tels) et par leur propension pour les réfutations sommaires? Ces facteurs interviennent peut-être à divers degrés et il serait dommage de les méconnaître. Ils ne constituent cependant pas une explication satisfaisante d'oppositions récurrentes. Il nous faut faire ici notre seconde remarque. C'est l'un des paradoxes du discours de justification de l'institutionnalisation de la philosophie que d'utiliser, pour se manifester, certains des modes tiaditionnellement réservés à l'activité théorique: l'écriture dans des revues disciplinaires et la tenue de colloques dont l'organisation se modèle sur ceux qui sont tenus par des sociétés savantes. Le paradoxe est avivé par le fait que les activités propres à favoriser l'énoncé du discours théorique sont relativement peu fréquentes dans notre milieu et que le temps que l'on peut y consacrer doit être conquis sur les horaires qui requièrent des investissements de plus en plus considérables de la part des professeurs. À témoin, ces exemples multiples où la production théorique d'un professeur de philosophie se réduit à quelques articles de circonstance sur «la fonction sociale de la philosophie». D’où le sentiment, parfois légitime, que le discours de justification usurpe la place du discours de vérité et peut en venir à constituer un alibi pour l'impuissance de la philosophie à parler d'autre chose que de son intérêt. Nous disions plus haut qu'il ne convenait pas de juger le discours de justification à l'aide de critères qui lui sont hétérogènes; une logique identique nous contraint maintenant de faire valoir que le discours de justification ne saurait se substituer à l'activité théorique, qu'il n'a pour fin que de permettre. Un groupe d'enseignants qui se bornerait à ne pouvoir parler que des raisons 
pour lesquelles on devrait le laisser exister est un groupe d'enseignants stériles. Lorsqu'il occupe tout l'univers du discours, c'est le propre du discours de justification que de se détruire.

1. Les justifications qui ont été apportées de la pratique institutionnalisée de la philosophie ont eu deux objets bien distincts : l'enseignement universitaire de la philosophie et l'enseignement collégial. La plupart des justifications récentes ont pour objet l'enseignement de la philosophie au niveau collégial ; c'est plutôt de ce type de justification dont nous nous préoccuperons, sans toutefois passer sous silence les justifications de l'enseignement universitaire.

La part des curricula d'études collégiales qui est consacrée à des enseignements de philosophie ayant été mise en cause par un projet de nouveau régime pédagogique, une part importante des textes qui tentaient de justifier l'opportunité d'un enseignement de philosophie au niveau collégial a été produite à l'époque où il fut question de déposer ce projet de nouveau régime pédagogique. Beaucoup de ces textes ont été rédigés par les responsables de la Coordination provinciale de l'enseignement de la philosophie, entre les années 1972 et 1974, et sont consignés dans les Cabiers pédagogiques de la Coordination provinciale de l'enseignement de la philosophie.

2. Il est, je crois, une même trame qui, avec des accents très divers, parcourt le type de justification qui a été donné de l'enseignement de la philosophie. Nous partirons du texte d'une conférence prononcée par le cardinal Villeneuve (alors évêque de Gravelbourg) devant les membres de l'Académie canadienne Saint-Thomas d'Aquin. Cette conférence porte sur le rôle de la philosophie à l'intérieur de l'université. Ce thème devait par la suite susciter de multiples textes et conférences de la part de l'institution philosophique québécoise. L'un des passages les plus significatifs de la conférence du cardinal Villeneuve se lit comme suit:

Je veux donc exposer maintenant comment, non seulement l'universitaire-philosophe, de ses hauteurs, ${ }^{*}$ doit pouvoir juger les sciences et les contrôler, mais d'une façon prochaine les

* Nous soulignons. 
stimuler et les diriger. En effet, notre philosophie, (..) éduque et forme le spécialiste ${ }^{1}$.

Le même thème est repris plus loin :

Or c'est ici que le rôle de la philosophie est régulateur dans la formation du spécialiste. Sans doute, la spécialisation est nécessaire mais non une spécialisation hâtive, fatalement stérile, non une spécialisation exclusive qui rend myope (...) Une Université a besoin de facultés distinctes, dont chacune se livre intensément à ses recherches propres de quelque aspect de la vérité et de la science. Mais, sous peine de tout compromettre, que le spécialiste commence par avoir l'esprit universel et qu'il continue de se tenir enraciné dans la métaphysique. Sans quoi, il fait une oeuvre morte et mortifère. Pour ne point la trouver dans son éprouvette, le chimiste niera l'âme...2

L'espace nous manque pour faire le commentaire approprié de ces textes qui eurent une influence considérable. J'en retiendrai trois choses: (i) la mise en place de la polarité entre la spécialisation et ce qui est désigné comme esprit universel; cette mise en place s'accompagne d'une valorisation du second membre de l'opposition; (ii) l'identification du lieu où se tient le philosophe et d'où il parle comme dehors et comme extériorité; la figure que prend dans le texte de Villeneuve l'extériorité est celle de la hauteur; (iii) la liaison de ce dehors ou de cette extériorité avec une axiologie; le dehors de la spécialisation est conçu dans le texte cité plus haut comme esprit universel qui se réalise sous les traits d'une métaphysique; or, comme le montre le passage relatif au chimiste qui nie la réalité de l'âme, le métaphysique se définit essentiellement comme un ensemble de v'aleurs. Il n'est, selon moi, pas un seul discours qui s'emploie à justifier l'institutionnalisation de la philosophie au Québec qui ne s'articule sur l'un ou l'autre de ces thèmes. Je dois cependant être ici explicite. Je n'affirme en rien que tout le discours de justification qui s'est énoncé par la suite a été effectivement influencé par ces textes du cardinal Villeneuve.

1. Villeneuve, J.-M.-R. (1930), «Le rôle de la philosophie dans l'oeuvre des universités catholiques», dans L'Académie canadienne Saint-Tbomas d'Aquin, Fondation, Première session (novembre 1930), Québec, 1932, pp. 203 à 262 , p. 221.

2. Villeneuve (1930), pp. 225-26. 
Il est, en effet, facile de constater que les thèmes que nous avons extraits ne lui appartiennent pas en propre. La question de l'appartenance ne doit pas être tranchée ici dans le sens de l'appartenance des thèmes à un individu qui aurait influencé tous les autres, mais, au contraire, dans le sens d'une appartenance commune d'un groupe d'individus à un même paradigme de justification qui transcende les auteurs et aliquel il est difficile d'assigner sans arbitraire une origine. Nous tenterons maintenant de montrer sommairement que le discours de justification de la philosophie s'est effectivement centré sur ces thèmes.

3. Une argumentation comme celle que présente le cardinal Villeneuve a faii une relative unanimité dans les milieux philosophiques québécois, complètement dominés par le clergé, jusqu'aux bouleversements de ce qu'on a appelé la révolution tranquille. $\bar{A}$ s'en rapporter aux discours publics de cette époque, on peut dire que l'éducation a constitué l'objet privilégié de la révolution tranquille. Le résultat le plus tangible de toutes ces remises en questions du système existant d'éducation a été la production du Rapport Parent, dont les recommandations ont donné naissance à la création du régime d'études collégiales, sous lequel, pour l'essentiel, nous vivons toujours. Or, il est banal de le rappeler, toute la conception des ćtudes collégiales qui s'exprime dans le Rapport Parent repose sur un ćquilibre souhaité entre la spécialisation des curricula et les impératifs d'une formation dite générale. La polariéé entre ce que Villeneuve appelait, d'une part, la spécialisation et, d'autre part, l'esprit universel est au coeur même du projer pédagogique qui s'énonce dans le Rapport Parent, et la philosophie y tire sa justification essentiellement de ce qu'elle est mise à contribution pour assurer la formation dite générale.

Pour répondre à ces exigences de formation générale et de spécialisation, les étudiants de l'institut devront avoir un programme d'études composé de trois types de cours: quelques. cours communs obligatoires pour tous, des cours de spécialité et des cours complémentaires à la spécialité. À ce niveaul, les cours communs obligatoires devront être réduits au strict minimum. Nous proposons ceux-ci: langue maternelle, langue seconde, éducation physique et philosophie. ${ }^{3}$

3. Rapport de la Commission royale d'enquête sur l'enseignement dans la Province de Québec (1964), Deuxième partie ou tome deux, section 274, quatrième édition, août 1965 , pp. 165-66, vol. 2 . 
La formation générale, telle que la conçoit le Rapport Parent peut être caractérisée par deux traits; (i) sa fonction est de créer un facteur d'unanimité parmi les futurs citoyens; (ii) elle consiste, en conséquence, en un certain nombre de contenus qui seront communs a tous les étudiants qui atteindront le niveau collégial.

4. Les justifications de l'enseignement de la philosophie qui seront produites, postérieurement à la déposition du Rapport Roquet, par la Coordination de l'enseignement de la philosophie vont consister, pour l'essentiel, à tenter de démontrer la nécessité d'une culture dite, dans la foulée du Rapport Parent, générale. L'enseignement de la philosophie étant lié de façon infrangible avec l'acquisition d'une culture générale, prendre parti pour la seconde équivaut alors à justifier la nécessité du premier terme de la relation. Quelques changements interviennent néanmoins dans la conception de la culture dite générale. Le plus important de ces changements est le suivant: la culture générale n'est plus conçue comme la possession d'un ensemble de contenus mais plutôt comme l'acquisition commune d'une attitude. Cette attitude est définie par un double mouvement de distanciation. et d'appropriation dans les cahiers pédagogiques de la Coordination provinciale de l'enseignement de la philosophie (voir, en particulier, le cahier d'octobre 1972).

5. Un texte devait avoir une grande influence sur les tentatives pour justifier l'enseignement de la philosophie. C'est le texte de Fernand Dumont et de Guy Rocher intitulé : "L'expérience des CEGEP, l'urgence d'un bilan », publié dans le numéro huit de la revue Critère. L'expression même de culture générale est mise en question, ainsi que la possibilité de transmettre aux étudiants un certain nombre de notions qui leur seraient communes. Cet article nous propose de substituer à la culture générale une culture dite fondamentale dont les caractéristiques sont les suivantes. La culrure fondamentale ne se définit pas par la possession d'un certain nombre de contenus mais par celle d'une habilité dont la caractéristique la plus remarquable est la transférabilité (sa fécondité peut être vérifiée dans plusieurs champs de connaissance). La culture fondamentale débouche sur un au-delà qui est nommé transcendance : 
Aussi faut-il qu'une éducation qui se prétend fondamentale, critique et transférable fasse appel à la transcendance. La transcendance, ce n'est pas nécessairement Dieu. Il suffit d'avoir la moindre connaissance de la pensée moderne pour éviter cette méprise. La transcendance, c'est plus simplement ce lieut éminent où l'on se tient * quand on fait lhistoire, quand on parle, quand on trace des théorèmes. Ce lieu bypothétique vaut êtrc exploré pour lui-même: c'est pourquoi nous croyons, mais dans ces limites, que l'enseignement de la philosopinie devrait faire partie d'une culture fondamentale. ${ }^{4}$

6. Cet article de Dumont et de Rocher a eu une grande influence. ${ }^{5}$ Leurs idées ont, pour l'essentiel, été reprises par la Coordination de l'enseignement de la philosophie, comme en témoigne le Cabier pédagogique d'avril 1973, qui présente le texte d'un colloque organisé par la Coordination de l'enseignement de la philosophie sur le thème de l'enseignement de la philosophie. Il est néanmoins un changement dans la conception de la culture générale ou fondamentale que vient à jour à ce colloque et qui prendra une importance considérable. Nous avons déjà dit que la culture générale se définissait dans les cahiers de la Coordination comme un double mouvement de distanciation et d'appropriation. De ces deux mouvements, c'est le moment négatif - celui de la distanciation - qui sera progressivement accentué. Le Mémoire an Conseil supérieur dé l'éducation, présenté par la Coordination provinciale en février 1974, propose de voir dans la philosophie une «interprétation et (une) critique des pratiques discursives qui entourent l'étudiant.» Ce terme de critique, prornis à une grande fortune, se trouvait déjà dans le texte de Dumont et Rocher.

7. Les traces écrites des justifications que donnent à l'enseignement de la philosophie les représentants de la plus grande modernité sont plus difficiles à trouver, ces jeunes professeurs ne faisant pas encore partie de «l'establishment» philosophique

* Nous soulignons.

4. Dumont, F. et Rocher, G., (1973), "L'expérience des Cegep: urgence d'un bilan ». Critère, no 8 (janvicr 1973), pp. 11 à 25, p. 21.

5. L'expression de culture fondamentale, avec un sens apparenté à celui que lui donnent Dumont et Rocher, apparaît dans le Rapport Nadeau, à la place de l'ancienne formation ou culture généralc. 
québécois. Néanmoins, il me semble que l'instance de la critique et de la nécessaire distance à prendre par rapport aux sollicitations des diverses idéologies tient une place prépondérante dans le rôle qui est attribué à l'enseignement de la philosophie. Elle rassemble des pratiques de la philosophie dont les allégeances idéologiques sont profondément divergentes.

8. La permanence de la triple thématique que nous avons distinguée à l'occasion du commentaire d'un texte du cardinal Villeneuve peut être aisément vérifiée. Jusqu'à l'apparition, relativement récente, de la définition de l'enseignement de la philosophie comme pratique critique, toute la question de la justification des enseignements de philosophie s'est élaborée dans l'horizon de la polarité spécialisation/formation générale, la philosophie étant associée, nous l'avons vu, aux requêtes d'une formation générale. Il faut insister sur le caractère profondément institutionnel de cette justification de la philosophie institutionnelle: le rôle de la Coordination provinciale de l'enseignement de la philosophie a consisté, pour l'essentiel, à défendre avec opiniâtreté le projet pédagogique institutionnalisé par le Rapport Parent. Il est peu d'arguments que l'on trouve dans les Cabier's pédagogiques de la Coordination provinciale qui soient autre chose qu'une reprise plus ou moins voilée ou consciente de ce qui se rencontre déjà dans le Rapport Parent.

Le troisième des thèmes que nous avons distingués - une définition axiologique de ce qui est posé comme dehors - se retrouve sous la forme d'urx plaidoyer en faveur de la transcendance dans le texte de Dumont et de Rocher.

Reste notre second thème: la détermination du lieu de la philosophie comme dehors. Nous désirons lui réserver un traitement à part, car c'est luị qui nous paraît apte à constituer une catégorie sous laquelle peut être subsumé tout le discours de justification qui s'est produit au Québec.

9. La catégorie que nous désirons introduire est celle de l'ektopie, que nous opposerons à celle de l'entopie. Ces deux termes, dont l'étymologie est transparente, suggèrent, pour le premier, un mouvement de retrait vers un dehors, une extériorité ou un hors-lieu et, pour le second, un mouvement d'insertion dans la direction contraire. Il m'a semblé, à la suite de la 
lecture de ce que je crois être le plus grand nombre des textes produits au Québec sur le sujet de la justification de l'enseignement de la philosophie, que le premier de ces termes était propre à donner un nom à la communauté, à mon avis profonde, qui unit tous ces textes, et que le second pouvait servir à nous rappeler que le style ektopique de justification n'était pas le seul possible. Style ektopique, qu'est-ce à dire? le couple ektopie/ entopie ne doit en aucune façon être confondu avec un autre couple qui pourrait paraitre son homologue, à savoir le couple négation/affirmation. J'entends, en gros, par ektopie tout mouvement de dégagement par rapport au donné historique, que celui-ci soit conçu sous sa forme matérielle ou sous sa forme idéologique. L'affirmation de valeurs abstraites (au sens non péjoratif de ce terme), qui débouche souvent sur la position d'une transcendance, et donc d'un hors-lieu, ou sur celle d'un accomplissement définitif de l'histoire, ressortit à un mouvement ektopique. Il en est de même d'une prise de position en faveur d'un type de formation qui serait propre à équilibrer, par les vertus de distanciation habituellement prêtées à une formation générale, les effets réputés néfastes de la trop grande coïncidence d'un individu avec sa spécialité professionnelle. La spécialisation professionnelle, il y a lieu de le noter, est précisément ce qui assigne à un individu son lieu tant économique que social. On est donc parfaitement justifié de qualifier d'ektopique une formation qui se donne pour fin de conjurer les suites de la spécialisation indue des personnes.

Ce que l'on affirme ici de la culture générale vaut a fortiori de l'attitude critique, qui constitue la figure pédagogique qui s'est substituée à l'idée de formation générale dans la justification de l'enseignement de la philosophie. L'ektopisme forme la réalité même du projet critique, comme en témoigne abondamment toute la métaphorie par laquelle il s'exprime et qui s'articule sur des notions comme celles de mise à distance, de décentrement, de recul etc. J'estime, pour ma part, qu'en dépit des apparences, la solidarité logique est suffisante entre un discours de justification qui s'organise autour de la polarité spécialisation/formation générale et un autre qui argue à partir de la nécessité d'impartir aux étudiants une attitude critique pour qu'on les subsume sous la même catégorie d'ektopie. Même 
si les valeurs défendues ne sont pas identifiques, l'un et l'autre discours se fonde explicitement sur une axiologie.

Nous essaierons, à la fin de cet article, de donner quelques exemples d'une conception entopique de la pratique de la philosophie.

10. La position que nous adoptons envers une pratique ektopique de la philosophie est, du moins en intention, essentiellement descriptive. C'est pourquoi nous n'aimerions pas que les quelques foyers de tension dont nous allons expliquer la présence au sein d'une tentative pour justifier une pratique ektopique de la philosophie fussent interprétés comme autant de raisons pour modifier au profit de l'entopie notre pratique de la philosophie. Il est une part d'ektopisme qui est irrémédiablement liée à la nature de la pratique philosophique. Une autre remarque: nous avons employé à dessein l'expression «foyers de tension » pour désigner les diffícultés dont nous allons faire état. Il n'est aucune de ces difficultés qui ait en notre esprit le statut d'une contradiction, au sens fort de ce terme. Ces difficultés existent à divers degrés et peuvent receroir dans la pratique une solution. Voici quels sont ces foyers de tension. Ils concernent davantage la philosophie comme projet critique que la philosophie comme formation générale. Les difficultés liées à cette idée n'ont plus besoin d'être soulignćes.

L'objet de la mise à distance, dans une pratique critique de la philosophie, est plus habituellement le donné culturel, tel qu'il se manifeste sous la forme d'un certain nombre d'idćologies, que le donné matériel comme tel. La difficulté inhérente à toute critique de la culture est qu'elle s'effectue toujours à partir de positions culturelles (idéologiques ou théoriques) qui sont extrêmement opérantes et parfois profondément sédimentées. D'où le caractère illusoire d'une mise à distance qui a pour condition la mise à l'abri d'un certain nombre de contenus et de thèses qui ne sont jamais questionnés et la duperie d'un décentrement de l'étudiant qui n'est que le prétexte à son recentrement. Le résultat pratique d'une pratique ektopique de la philosophie, assez inconciliable avec les objectifs qu'elle estime poursuivre, est trop souvent de conférer le statut d'un non-lieu à l'endroit d'où elle parle. Ce non-lieu n'est par définition 
jamais décrit ni interrogé. Les voix venues de nulle part sont toujours celles qui se font entendre avec le plus d'insistance.

La difficulté précédente reçoit une traduction immédiate sur le plan institutionnel. Lorsqu'une instance institutionnalise le discours qui se donne pour fonction de la critique ou lorsque, de façon converse, la critique se laisse intégrer à l'institution qu'il prend habituellement pour cible, il est difficile de trancher de quel côté se trouve l'ingénuité (qu'il y ait naïveté de la part de l'une au moins des parties en cause est incontestable). De la même façon qu'il y a une difficulté à penser qu'une délimitation rigoureuse du culturel s'effectue à partir de positions qui intègrent une tradition culturelle profondément sédimentée, on conçoit mal que ceux-là qui ont des intérêts dans l'existence de l'institution - - c'est cela d'abord que d'être institutionnalisé - la mettront profondément en cause. La position de repli étant elle-même à l'intérieur du périmètre que trace l'institution, s'y retirer ne peut avoir pcur résultat qu'une institutionnalisation accrue, puisqu'elle cesse alors d'être consciente d'elle-même.

Une troisième difficulté, liée à la précédente, est la suivante. Il est manifeste que l'aspect critique de la pratique philosophique sera diversement accentué selon les destinataires du discours de justification. Il est clair qu'un mémoire adressé au ministère de l'Éducation aura un ton assez différent de celui d'un tract adressé aux "étudiants. On aurait grandement tort de méconnaitre les effets de ces divers ajustements du discours de justification, qui est, rappelons-le, un discours de circonstance. Comme, au Québec, le discours de: justification s'est arrogé la place du discours théorique, les prédicats du discours stratégique sont devenus associés à ceux du discours théorique et grèvent de façon marquée sa prétention à constituer un discours de vérité.

Il me semble, en dernier lieu, que leur position critique face à l'institution a conduit les professeurs à projeter à l'extérieur d'eux-mêmes la menace qui pèse sur la philosophie telle qu'elle est actuellement institutionnalisée. Toute tentative pour réduire la place des enseignements de philosophie est souvent perçue sous les traits d'une réaction défensive de la part de l'autorité politique. Il y a là, me semble-t-il, une surestimation de la part 
de la philosophie des effets de sa critique, à laquelle il y aurait profit à renoncer à l'avantage d'une évaluation rigoureuse de la qualité des enseignements de philosophie qui sont donnés et de la réception qui est faite à ces enseignements par les étudiants. Je crois, pour ma part, que si les enseignements de philosophie faisaient l'unanimité chez les étudiants, la menace qui pèse sur ces enseignements serait beaucoup moins pressante. Je serais à cet égard curieux de connaitre les résultats d'une enquête rigoureuse (qui ne présumerait pas d'avance des réponses) organisée par la Coordination provinciale de l'enseignement de la philosophie sur l'opinion des étudiants sur l'enseignement de la philosophie. L'idée que se font actuellement les professeurs de philosophie de tout niveau au sujet de l'accueil qui est fait à leurs enseignements m'apparaît trop souvent marquée au coin de la plus large complaisance.

11. J'aimerais, en terminant, présenter quelques exemples d'une pratique de la philosophie qui serait davantage entopique - située.

Une première façon pour la philosophie d'être entopique serait de renouer avec l'instance de la doctrine. La différence entre la critique et la doctrine, à tout le moins au niveau d'une pratique de l'enseignement, est que la première vise avant tout à provoquer une prise de conscience alors que la seconde ambitionne de transmettre une connaissance. Je ne crois pas, pour ma part, qu'un enseignement qui renonce à communiquer une connaissance soit appelé à durer. La tragédie intellectuelle québécoise est, à la suite d'une expérience traumatique de l'orthodoxie doctrinale, d'avoir confondu la doctrine avec le dogme. Cette confusion n'est en rien légitime. Il est actuellement impérieux de rappeler à l'enseignement de la philosophie qu'une philosophie n'est tien si sa méditation sur le doute n'est pas suivie de la découverte d'un équivalent du cogito. Nous n'aurions aucune mémoire du cartésianisme si la première Méditation n'avait pas eu de suite.

Mais y a-t-il en philosophie des connaissances suffisamment assurées pour qu'on puisse les transmettre aux étudiants, avec la certitude de leur communiquer des résultats acquis? Il peut certainement s'en trouver. Un champ où se recontre habituellement un ensemble de connaissance positives en philosophie est 
celui de l'histoire de cette discipline. Nous ne formulons pas ici le souhait banal et réactionnaire d'un retour des enseignements à l'histoire traditionnelle de la philosophie. Je ne laisse cependant pas de m'étonner de l'absence en philosophie d'enseignements relatifs à notre histoire intellectuelle québécoise $\mathrm{Si}$ l'on pense, comme il faut le faire, que la condition de la rigueur de la critique est la pleine connaissance de son objet, on peut douter du sérieux de toutes les critiques de l'idéologie québécoise qui s'énoncent actuellement. La description précise de cette idéologie, ainsi que la production de son histoire, restent à faire. Ces tâches constituent de bons exemples d'une pratique que je qualifierais d'entopique de la philosophie et son enseignement.

Il est un autre champ de la philosophie où les connaissances font une relative unanimité. Ce champ est celui de la méthodologie et, en particulier, celui de la logique. C'est, je crois, une erreur que d'assimiler les propositions d'ordre méthodologique ou logique que peut énoncer la philosophie à une critique de la connaissance. Cette erreur ressortit, entre autres facteurs, à un oubli que l'objet de la critique kantienne - la raison pure -.. n'est pas l'instance qui produit la connaissance (la science) mais celle qui ambitionne de faire la métaphysique; postérieurement à Kant, l'épistémologie a été plus ou moins confondue par les faiseurs de manuels avec une «critique de la connaissance», sans que l'on s'interroge sur le sens des termes employés dans cette expression. Mais la question kantienne, lorsqu'elle porte sur la science et non sur la métaphysique, n'est pas celle de la critique et de l'évaluation mais bien au contraire celle des conditions de possibilité d'unc science dont les résultats, on l'a souvent remarqué, ont été acceptís par Kant de manière non critique. Il m'apparaît opportun, avant de donner à nos étudiants des procédures pour évaluer les connaissances, de les instruire sur la manière de les produire. Possédant de façon plus ou moins adéquate des moyens souvent arvancés d'évaluer et de critiquer des connaissances qu'ils n'ont jamais appris à produire, les étudiants en sont la plupart du temps réduits à passer au crible de la critique les propositions qu'ils ont, avec des fortunes diverses, tenté de s'extraire et qui dépassent rarement le niveau intuitif ou le lieu commun culturel. L'apprentissage inévitable qu'il ne manquent pas, chaque fois, de faire est celui de l'inadé- 
quation des connaissances qu'ils ont tenté de produire. Cet écart renouvelé entre l'instrumentation critique et l'outillage méthodologique ne peut, à la longue, qu'engendrer le scepticisme et la démotivation par rapport à l'esprit de recherche.

Université du Québec à Montréal 